\title{
Ciclo de produção de cogumelos comestíveis cultivados em resíduos lignocelulósicos da fruticultura Amazônica: Um estudo de caso.
}

\section{Production cycle of edible mushrooms grown in lignocellulosic residues from Amazonian fruit growing: A case study}

\author{
Maria do Perpétuo Socorro de Lima Verde Coelho ${ }^{1 *}$, Adrya da Silva Figueiredo ${ }^{1}$, Salomão
} Rocha Martim$^{1}$, Maria Francisca Simas Teixeira ${ }^{2}$

\begin{abstract}
RESUMO
Alimentos saudáveis com propriedades terapêuticas está impulsionando a produção e a comercialização de espécies de Pleurotus. Em escala comercial, esses cogumelos são cultivados em matriz sólida, utilizando diferentes substratos orgânicos. Resíduos lignocelulósicos disponíveis na Amazônia vêm demonstrando potencial para obtenção de basidiomas de Pleurotus. Este trabalho apresenta contribuições científicas promissoras que demonstram os ciclos de produção de Pleurotus ostreatoroseus e $P$. albidus, cultivados em resíduos da fruticultura amazônica pela tecnologia da fermentação no estado sólido. Essas espécies desenvolveram a fase micelial completa em grãos de trigo (spwan), em 15 dias e cinco dias, respectivamente. Porém, demonstraram diferentes ciclos produtivos quando cultivadas em exocarpo de cupuaçu misturado com farelo de arroz. P. ostreatoroseus apresentou dois ciclos de produção. No entanto, P. albidus foi observado apenas um fluxo produtivo, caracterizado pela maturação e colheita dos basidiomas. Resíduos lignocelulósicos da fruticultura amazônica são substratos eficientes para a produção de P. ostreatoroseus e P. albidus.
\end{abstract}

Palavras-chave: Basidioma; Fermentação em estado sólido; Pleurotus albidus; Pleurotus ostreatoroseus; Theobroma grandiflorum.

\section{ABSTRACT}

Healthy food with therapeutic properties is boosting the production and commercialization of Pleurotus species. On a commercial scale, these mushrooms are grown in a solid matrix, using different organic substrates. Lignocellulosic residues available in the Amazon have shown potential for obtaining Pleurotus basidiomes. This work presents promising scientific contributions that demonstrate the production cycles of Pleurotus ostreatoroseus and P. albidus, cultivated in residues of the Amazonian fruit culture by the solid state fermentation technology. These species developed the complete mycelial phase in wheat grains (spwan), in 15 days and five days, respectively. However, they showed different production cycles when cultivated in cupuaçu exocarp mixed with rice bran. P. ostreatoroseus showed two production cycles. However, P. albidus was observed only one productive flow, characterized by the maturation and harvest of the basidiomes. Lignocellulosic residues from Amazonian fruit crops are efficient substrates for the production of P. ostreatoroseus and P. albidus.

\footnotetext{
${ }^{1}$ Rede de Biodiversidade e Biotecnologia da Amazônia Legal (REDE BIONORTE) *E-mail: mcoelho@ufam.edu.br

${ }^{2}$ Universidade Federal do Amazonas.
} 
Keywords: Basidioma; Solid state fermentation; Pleurotus albidus; Pleurotus ostreatoroseus; Theobroma grandiflorum.

\section{INTRODUÇÃO}

Os cogumelos são universalmente reconhecidos como alimentos e vem sendo amplamente cultivados em escala comercial, em diversos países. Espécies de cogumelos comestíveis têm despertado interesse socioeconômico, não só por constituírem um pool de nutrientes (proteínas, carboidratos, minerais, vitaminas, aminoácidos essenciais, baixo teor de gorduras e fibra dietética), mas também por sintetizarem compostos antimicrobianos, antioxidantes, antitumoral, anticancerígeno, antiviral, anti-inflamatório, entre outros, que os caracterizam como alimentos funcionais (BEHERA; GUPTA, 2015; BONONI; GIMENES; ROJAS, 2015).

Espécies de Pleurotus que são produzidas em pequena e grande escala, em muitos países, ocupam a terceira posição na produção de cogumelos comestíveis (GOGAVEKAR, et al. 2014; CORRÊA et al., 2016; ADEBAYO; ALOKE, 2017). Pleurotus ostreatoroseus e P. albidus que estão disponíveis no mercado, geralmente são colhidos de ecossistemas florestais de clima tropical e subtropical onde constituem agentes de degradação de diversos resíduos lignocelulósicos (MARTIM et al., 2017; GAMBATO et al., 2018; SILVA et al., 2021).

Com base no hábito de vida dos cogumelos, a produção envolve um conjunto de processos que viabilizam a conversão de resíduos lignocelulósicos em alimentos de alta qualidade nutricional (ATILA, 2016). Neste contexto, a produção de cogumelos está alinhada com os conceitos atuais de economia circular, modelo econômico voltado para o uso eficiente de recursos por meio da minimização de resíduos e ciclos fechados de produtos (MORSELETTO, 2020). Além disso, o cultivo de cogumelos para fins comerciais auxilia na proteção de recursos naturais e gera benefícios socioeconômicos que podem contribuir para o desenvolvimento regional sustentável (MEYER et al., 2020).

A fermentação no estado sólido é uma tecnologia eficiente e barata para produção de cogumelos, pois neste bioprocesso são utilizados resíduos lignocelulósicos como substrato para o crescimento dos fungos comestíveis (ARAUJO et al., 2016; ZIED; IOSSI, 2018). Entretanto, para atender à demanda crescente por cogumelos comestíveis e promover o desenvolvimento econômico, em diversos países, pesquisas são realizadas 
para aprimoramento de técnicas de produção, associadas ao uso ambientalmente correto de substratos da fruticultura regional (DE ITA et al., 2015; MKHIZE et al 2017).

No Brasil, em especial na região Norte, substratos da fruticultura amazônica vêm sendo utilizados nos cultivos em escala laboratorial, para avaliar e otimizar o ciclo produtivo de diferentes espécies de cogumelos com potencial econômico. Exocarpo de cupuaçu misturado com casca de arroz (Oryza sativa L.) foram eficazes para produção de Lentinus citrinus e $P$. ostreatus, evidenciando a formação basidiomas de excelente valor nutricional (TEIXEIRA; PALHETA; CASTILLO, 2011; NEVES, 2014)

Com base no exposto, este trabalho tem por objetivo demonstrar os dados dos ciclos de produção de Pleurotus ostreatoroseus e Pleurotus albidus, cultivados em resíduos lignocelulósicos da fruticultura amazônica, como um processo ecoamigável para produção de alimentos alternativos e com qualidade nutricional.

\section{MATERIAL E MÉTODOS}

Nesta pesquisa foi realizado levantamento bibliográfico de acesso público compreendendo dados relativos ao uso de resíduos lignocelulósicos da fruticultura amazônica e agroindustrial disponíveis em artigos, dissertações e teses desenvolvidas no Laboratório de Micologia Industrial e Médica, ambiente físico pertencente à Coleção de Culturas DPUA, da Universidade Federal do Amazonas-UFAM. Nesses trabalhos científicos foram considerados dados que mostraram, desde a aquisição dos substratos, viabilidade, as estratégias e o respectivo ciclo de produção de Pleurotus ostreatoroseus e P. albidus, ambos do acervo da Coleção de Culturas DPUA.

\section{RESULTADOS E DISCUSSÃO}

Comumente, Pleurotus são cultivados em condições ambientais controladas, com a finalidade do conhecimento do ciclo de vida, preservação ambiental, reutilização e/ou no reprocessamento de materiais para desenvolvimento de tecnologias ecoamigáveis (PALHETA et al., 2011; SOUZA et al., 2016). Estas características biotecnológicas atendem aos requisitos de valorização de resíduos lignocelulósicos, além de auxiliarem no desenvolvimento econômico sustentável exigidos pela economia circular moderna (GRIMM; WÖSTEN, 2018; PIMENTA et al., 2021).

Embora tenham valor econômico, gastronômico e medicinal, os cogumelos não estão incluídos como produto alimentício integrante da dieta da população brasileira. Inclusive, a busca por fungos está aumentando, mas em muitas regiões há carência de 
empresas produtoras e, quando disponível no comércio importador, os altos preços desses produtos dificultam o acesso pela população (AZEVEDO et al., 2014; GAMBATO et al., 2018; CABRERA et al., 2020).

Em ecossistemas florestais, cogumelos crescem em resíduos vegetais, condição que favorece a colonização de folhas, frutos, sementes, caules, entre outros. Essa capacidade de adaptação em diversos substratos lignocelulósicos favorece o cultivo desses fungos comestíveis em condições padronizadas in vitro (FONSECA et al., 2015; PANDEY et al., 2020). Desse modo, estudos realizados com P. ostreatoroseus DPUA 1720 e P. albidus DPUA 1692, foi verificada a viabilidade da produção em substratos lignocelulósicos da fruticultura amazônica, usando a tecnologia de fermentação no estado sólido, bioprocesso observado em dois ciclos, conforme apresentado na figura 1 .

Figura 1: Fluxograma do ciclo de produção de espécies de Pleurotus

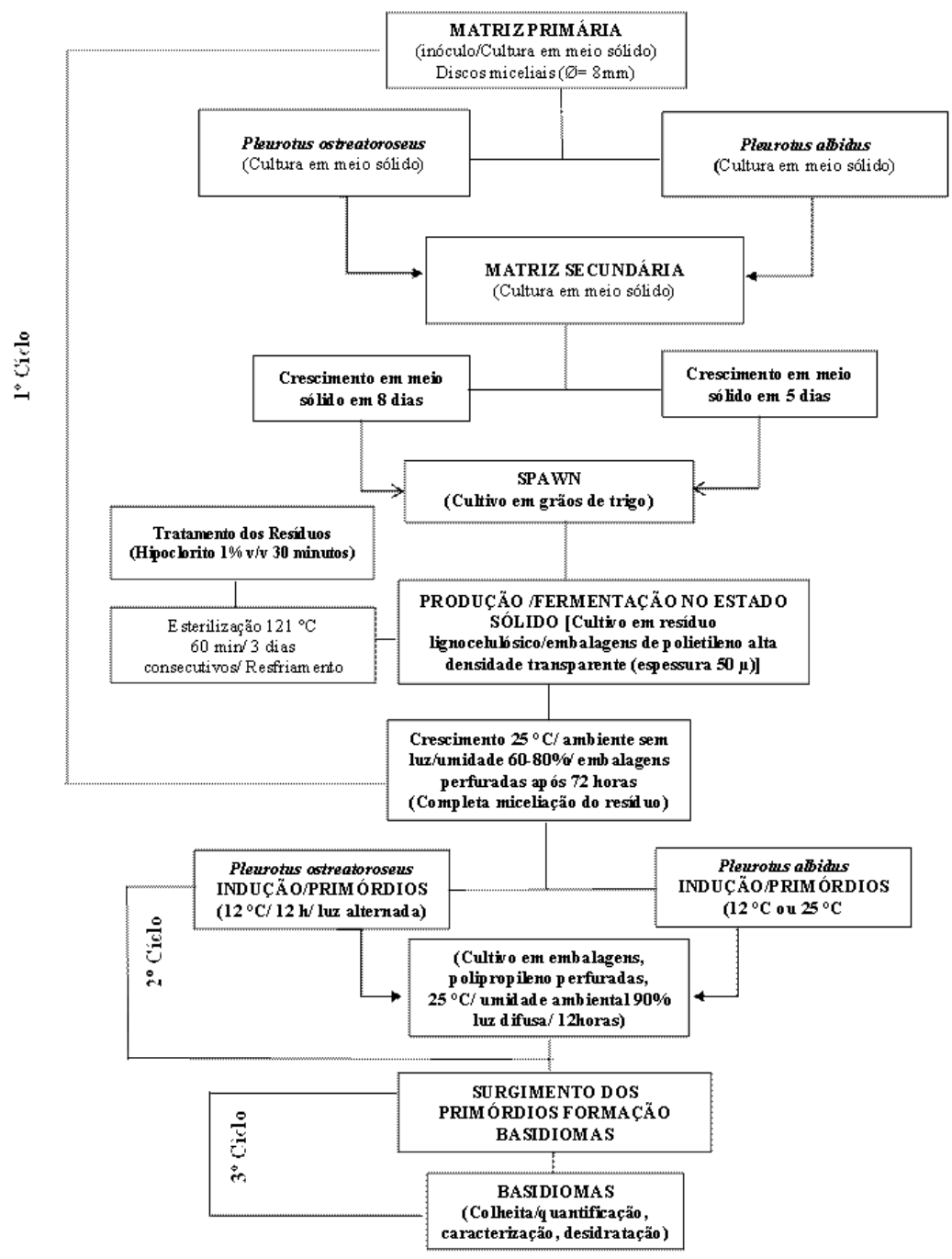

Fonte: Fonseca et al. (2014); Pimenta et al., (2021) e Barbosa et al., (2021). 
Os resultados de uma pesquisa motivadora, que promoveu a geração de novos estudos e oportunidades bioeconômicas, mostraram a viabilidade da cadeia tecnológica produtiva de $P$. ostreatoroseus isolado do bioma amazônico. Nessa produção orgânica, realizada por Fonseca et al., (2014), no início do ciclo de crescimento, P. ostreatoroseus desenvolveu a fase micelial completa em grãos de trigo (spwan), em 15 dias. Após inoculação do spawn em exocarpo de cupuaçu misturado com farelo de arroz [80:20 (\% $\mathrm{p} / \mathrm{p}$ )], foi verificado o surgimento dos primórdios em três dias, seguido do crescimento e maturação dos basidiomas. A primeira colheita que caracterizou o primeiro fluxo foi feita aos 23 dias de cultivo (Figura 2) e, em 42 dias, o segundo fluxo. Ao término da colheita, os basidiomas foram desidratados em condições adequadas para a manutenção de suas propriedades nutricionais e funcionais.

Figura 1 - Produção de P. ostreatoroseus DPUA 1720: (A) em exocarpo de cupuaçu+farelo de arroz (CC+FA com grãos de trigo; (B) Surgimento dos primórdios; (C) Primórdios e início do crescimento dos basidiomas; (D) Basidiomas maduros (E) $=($ Primeiro Fluxo $)$.

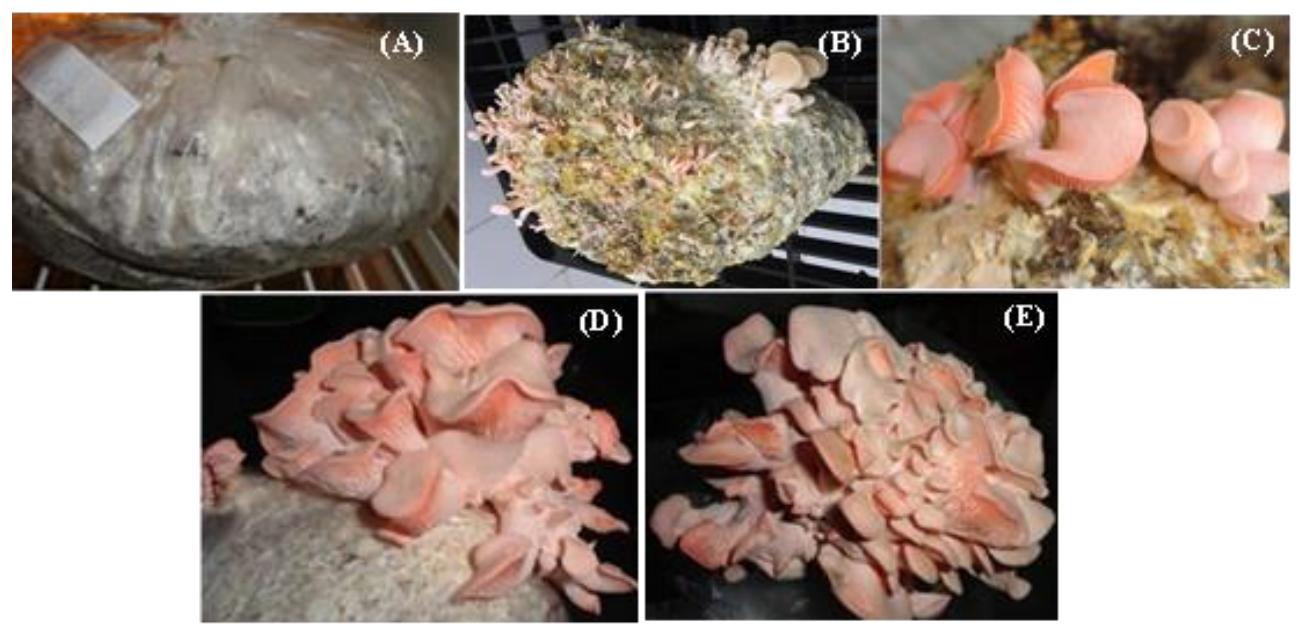

Fonte: Fonseca et al. (2014); Fotos da Coleção DPUA/UFAM

A Figura 2 mostra também que P. ostreatoroseus possui basidiomas de superficie lisa e coloração rósea característica dessa espécie. Barbosa, E. et al. (2021) relataram características similares, além de odor agradável e textura firme dos basidiomas. Para essa espécie, nas condições de cultivo, a estimativa de produção, com base na eficiência biológica, foi 22,90\%, em média. Eficiência biológica é uma variável que mostra a capacidade do cogumelo converter o substrato em basidiomas (corpo de frutificação) (CHANG e MILES, 2004; OLIVEIRA et al., 2007). Valor de eficiência biológica de aproximadamente $20 \%$ revela que se trata de substrato recomendável para 
uso no cultivo de cogumelo comestível, além de mostrar que, para cada $100 \mathrm{~kg}$ de substrato úmido, a produtividade pode abranger $20 \mathrm{~kg}$ de cogumelos in natura (STEFFEN et al., 2020). Na mistura de substrato formulado com exocarpo de cupuaçu, a biomassa de $P$. ostreatoroseus foi caracterizada como um produto alimentício com teor significativo de proteína e fibra, baixo teor de de lipídio, podendo ainda fornecer sais minerais e aminoácidos (FONSECA, 2013).

Pleurotus albidus é outra espécie que vem sendo investigada em relação aos aspectos fisiológico, biotecnológico e nutricional. Nos cultivos em resíduo lignocelulósico, em condições ambientais controladas, foram observados a colonização do substrato e o ciclo de produção de P. albidus. O spwan totalmente miceliado foi obtido em cinco dias e inoculado nos seguintes resíduos da fruticultura amazônica: exocarpo de cupuaçu misturado com farelo de arroz [80:20 (\% p/p)]. Em cinco dias, foi observada a miceliação total do substrato e, após choque térmico a $12{ }^{\circ} \mathrm{C}$, formação dos primórdios, em seis dias. O início do desenvolvimento dos basidiomas ocorreu aos nove dias de cultivo e com 15 dias a maturação e colheita dos basidiomas (Primeiro Fluxo), Figura 3. A análise de composição centesimal evidenciou que, entre os nutrientes de P. albidus, predominou o conteúdo de proteínas $(33,31 \mathrm{~g} / 100 \mathrm{~g}$ de produto).

Figura 2 - Produção de P. albidus DPUA 1692: (A) em exocarpo de cupuaçu+ farelo de arroz (CC+FA), inoculado com spwan preparado com grãos de trigo; (B) Surgimento dos primórdios; (C) crescimento dos basidiomas; (D) Basidiomas maduros (E) (Primeiro Fluxo).
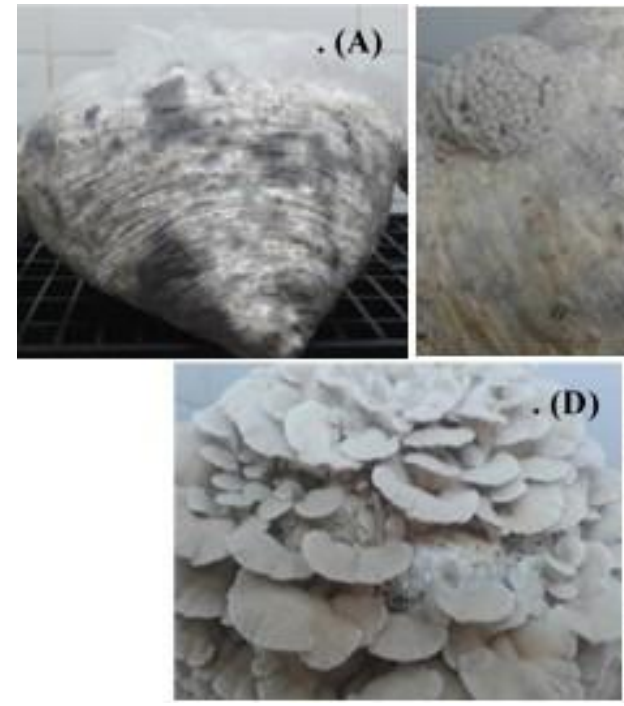

(D)

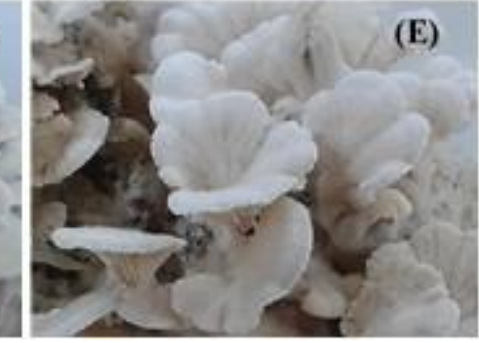

(E)

Fonte: Fotos do Coleção da Coleção DPUA/UFAM.

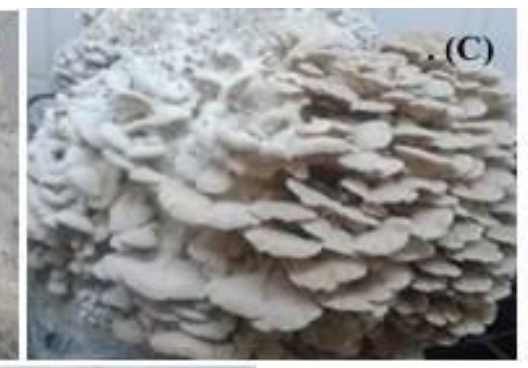


Nas condições avaliadas, $P$. albidus demonstrou crescimento rápido, invasor e excelente viabilidade para a reprodução, porém a eficiência biológica foi baixa $(5,33 \%)$. A eficiência biológica inferior à desejável, para a produção em escala industrial, está associada ao quantitativo de basidiomas desenvolvidos, condição que dificulta o crescimento dessas estruturas. Embora P. albidus não tenha apresentado crescimento eficaz in vitro, foi observado ciclo de produção completo. A partir destes resultados, outros estudos estão em andamento, para a identificação das condições de cultivo adequadas, para a produção em grande escala, utilizando resíduos da fruticultura amazônica.

Em um estudo pioneiro realizado por Kirsch et al., (2016), usando a tecnologia do cultivo em meio líquido, foi verificado que fonte de carbono e nitrogênio, além do pH, volume de inóculo e velocidade de agitação, são fatores que comprometem significativamente a produção de biomassa $P$. albidus.

Dados da literatura já comprovaram sua importância biotecnológica como fonte de diversos compostos bioativos. Souza et al. (2016) cultivaram P. albidus em casca de abacaxi e concluíram que a biotransformação desse resíduo resulta em um bioproduto com teores significativos de fibras $(14,23 \%)$, proteínas $(8,61 \%)$ e minerais $(5,26 \%)$, que pode ser utilizado para aumentar o valor nutricional e biológico de produtos de panificação.

Teixeira e Martim (2017) por processos tecnológicos concluiu que P. albidus sintetiza proteases coagulantes do leite bovino com características bioquímicas adequadas para uso na fabricação de queijo. Atualmente, o processo de produção e extração de coagulante do leite bovino utilizando $P$. albidus consta no depósito de pedido nacional de patente $\mathrm{n}^{\circ} \mathrm{BR} 1020170146723 \mathrm{~A} 2$.

Em outros experimentos realizados com exocarpo de cupuaçu com farelo de arroz, $P$. albidus foi uma espécie que sintetizou peptidases ou proteases ácidas, com predomínio de metalo e cisteíno peptidases, características que revelam aplicabilidade na indústria de alimentos (PIMENTA et al., 2021).

\section{REFERÊNCIAS}

ADEBAYO, E. A.; OLOKE, J. K. Oyster mushroom (pleurotus species); a natural functional food. Journal of Microbiology, Biotechnology Food Sciences, [S. L.], v.7, n.3, p. 254-264, 2017. 
ARAUJO, W.J. et al. Análise de custo da produção de champignon: estudo em uma propriedade rural no município de Domingos Martins-ES. Revista Científica Intelletto, v.1, n.1, p. 102-114, 2016.

ATILA, F. Effect of Different substrate Disinfection Methods on the Production of Pleurotus ostreatus. Journal of Agricultural Studies, v.4, n.4, 2016.

AZEVEDO, D. B.; OSORIO, R. M. L.; CARVALHO, R. Q. C.; CARDOSO, B. B. C. Cadeia de produção do cogumelo orgânico: o estudo de caso da empresa cogumelos brasilienses. Revista de Gestão Ambiental e Sustentabilidade - GeAS, v. 3, n.1. Jan./ Abr 2014.

BARBOSA, E. E. P. Biorrefinaria: exploração sustentável de resíduos lignocelulósicos para produção de cogumelo e bioproduto comestível. 2021. Dissertação (Mestrado em Biotenologia) Universidade Federal do Amazonas, Manaus:UFAM/PPGBiotec, 2021. (Apresentação em 29/07/2021)

BARBOSA, E. E. P.; PIMENTA, L.; BRITO, A. K. P.; MARTIM, S. R.; TEIXEIRA, M. F. Cultivo de cogumelo comestível em resíduos lignocelulósicos de floresta tropical para produção de proteases. Brazilian Journal of Development. Curitiba, v. 6, n. 11, p.92475-92485, nov. 2020.

BEHERA, S.; GUPTA, N. Utilization of vegetable waste for biomass production of some wild edible mushroom cultures. An International Journal Tropical Plant Research, v.2, n.1, p.: 05-09. 2015.

BONONI, V. L. R.; GIMENES, L.J.; ROJAS, A.C.B. Produção de cogumelos e biotecnologia. In: SIMPÓSIO INTERNACIONAL DE COGUMELOS NO BRASIL. 8., 2015. Anais[...] Sorocaba/SP: SICG, 2015. p. 96-109. Disponível em: https://uniso.br/publicacoes/2015-anais-sicog/sicog-versaoonline.pdf. Acesso em: 05 jun 2018.

CABRERA, L. C.; CONSTANTINO, L. V.; ANTUNES, P. S.; GONÇALVES, L. S. A.; CORTE, L. E. Caracterização da produção de cogumelos comestíveis: um estudo de caso na região de Londrina, Paraná. Pesquisa Sociedade e Desenvolvimento, v.9, n.7, 2015. (Ciências Agrarias e Biológicas)

CASTILlO, T. A.; PEREIRA, J. R. G.; ALVES, J. M.; TEIXEIRA, M. F. S. Mycelial Growth and Antimicrobial Activity of Species of Genus Lentinus (Agaricomycetes) from Brazil. International Journal of Medicinal Mushrooms, v.19, n. 12, p.1135-1143, 2018.

CHANG, S; MILES, G.P. Cogumelos: cultivo, valor nutricional, efeitos medicinais e impacto ambiental. Boca Raton, FL: CRC Press, 2004.

CORRÊA, R.C.G. et al. Uso biotecnológico, nutricional e terapêutico de Pleurotus spp. (Cogumelo-ostra) relacionado com a sua composição química: Uma revisão sobre os resultados da década passada. Trends in Food Science \& Technology, [S. L.], v.50, p. 103-117, 2016.

FONSECA, T. R. B. Pleurotus ostreatoroseus DPUA 1720: Avaliação do crescimento, produção de basidioma e determinação da atividade proteolítica em 
resíduos agroindustriais. 2013. 77 f. Dissertação (Mestrado em Biotecnologia) Universidade Federal do Amazonas, Manaus, UFAM, 2013.

FONSECA, T. R. B.; BARRONCAS, J. F.; TEIXEIRA, M. F. S. Produção em matriz sólida e caracterização parcial das Proteases de cogumelo comestível da floresta Amazônica. Revista Brasileira de Tecnologia Agroindustrial, v. 8, n. 1, p. 12271236, 2014.

FONSECA, T. R. B. et al. Cultication and nutritional studies of na edible mushroom from North Brazil. African Journal of Microbiology Research, v.9, n.30, p.1814$1822,2015$.

GAMBATO, G.; PAVÃO, E. M.; CHILANTI, G.; FONTANA, R. C.; SALVADOR, M.; CAMASSOLA, M. Pleurotus albidus Modulates Mitochondrial Metabolism Disrupted by Hyperglycaemia in EA. hy926 Endothelial Cells. BioMed Research International, [S. L.], v.2018, p.1-10, 19 jun.2018.

GOGAVEKAR, S.S. et al. Important nutritional constituents, flavour components, antioxidant and antibacterial properties of Pleurotus sajor-caju. Journal Food Science Technology, v.51, n.8, p. 1483-1491, 2014. Disponível em: DOI 10.1007/s13197-0120656-5. Acesso em: 02 jul. 2019.

GRIMM, D.; WÖSTEN, H. A. B. Mushroom cultivation in the circular economy. Applied Microbiology and Biotechnology, v. 102, n.18, p.7795-7803, 2018.

ITA, M. A. V.; ARANDA, D. P. B.; LEZAMA, C. P.; REYES, J. R. T.; MARTINEZ, A. I.; ROMERO-ARENAS, O. Evaluation of Substrates in the Elaboration of Secondary Inoculum for the Cultivation of Pleurotus ostreatus. Journal of Pure and Applied Microbiology, v. 12, n. 2, p. 679-686, jun. 2018.

KIRSCH, L. S.; MACEDO, A. J. P.; TEIXEIRA, M. F. S. Production of mycelial biomass by the Amazonian edible mushroom Pleurotus albidus. Brazilian Journal of microbiology, v.47, p. 658-664, 2016.

MARTIM, S. R.; SILVA, L. S. C.; ALECRIM, M. M.; SOUZA, B. C.; OLIVEIRA, I. M. A., TEIXEIRA, M. F. S. Proteases ácidas de cogumelo comestível da Amazônia para aplicabilidade industrial. Boletim Museu Paraense Emílio Goeldi. Ciências Natural. Belém, v. 12, n. 3, p. 353-362, set.-dez. 2017.

MEYER, V; BASENKO, E. Y; BENZ, J. P.; BRAUS, G. H.; CADDICK, M. X.; CSUKAI, M.; VRIES, R. P. de; ENDY, D; FRISVAD, J. C.; GUNDE-CIMERMAN, N. Growing a circular economy with fungal biotechnology: a white paper. Fungal Biology And Biotechnology, [S. L.], v. 7, n. 1, p. 1-55, 2 abr. 2020.

MKHIZE, S. S.; ZHARARE, G. E.; BASSON, A. K.; MTHEMBU, M. S.; CLOETE, J. Desempenho do cogumelo Pleurotus pulmonarius cultivado em resíduos de colmo de milho suplementado com vários níveis de farinha de milho e farelo de trigo. Food Science Technology, Campinas, v. 37, n. 4, pág. 570-577, dez. 2017.

MORSELETTO, P. Targets for a circular economy. Resources, Conservation \& Recycling, v.153, 104553, 2020. https://doi.org/10.1016/j.resconrec.2019.104553 
NEVES, K. C. S. Produção de proteases coagulante por espécies de Pleurotus de resíduos vegetais da Amazônia. Tese (Doutorado em Biotecnologia) Rede Nordeste de Biotecnologia - RENORBIO. Recife: UFPE, 2014.

OLIVEIRA, M. A.; DONEGA, M. A.; PERALTA, R. M.; SOUZA, C. G. M. Produção de inóculo do cogumelo comestível Pleurotus pulmonarius (Fr.) Quélet - CCB19 a partir de resíduos da agroindústria. Ciência Tecnologia de Alimentos. Campinas, v. 27(supl.), p.84-87, ago. 2007.

PALHETA, R. A.; VIEIRA, J. N.; NEVES, K. C. S.; TEIXEIRA, M. F. S. Crescimento micelial vertical de duas espécies de Pleurotus em resíduo agroindustrial da Amazônia utilizando planejamento fatorial. Caderno de Pesquisa, Série Biologia, v. 23, n. 3, p. 52-60, 2011.

PANDEY, A. K.; RAJAN, S.; SARSAIYA, S.; JAIN, S. K. Mushroom for the National Circular Economy. International Journal of Scientific Research in Biological Sciences, v.7, n. 6, p.58-66, dec. 2020.

PIMENTA, L.; BARBOSA, E. E. P.; BRITO, A. K. P.; MARTIM, S. R.; TEIXEIRA, M. F. S. Processo eco-amigável para selecionar substrato Lignocelulósico para produção de peptidases ácidas. Brazilian Journal of Development, Curitiba, v.7, n.1, p.3469-3479 Jan. 2021.

SILVA, S. D. da; SANTOS, E. C. dos; SILVA, W. M. C. da; MAGALHÃES, A. A. da S; BARBOSA, E. E. P; TEIXEIRA, M. F. S; PEREIRA, J. O; MOTA, A. J. da. Draft Genome Sequence of the Wild Edible Mushroom Pleurotus ostreatoroseus DPUA 1720. Microbiology Resource Announcements, [S. L.], v. 10, n. 4, p. 1-6, 28 jan. 2021.

SOUZA, R. A. T.; FONSECA, T. R. B.; KIRSCH, L. S.; SILVA, L. S. C.; ALECRIM, M. M.; CRUZ FILHO, R. F.; TEIXEIRA, M. F. S. Nutritional composition of bioproducts generated from semi-solid fermentation of pineapple peel by edible mushrooms. African Journal of Biotechnology, v.15, n. 12, p. 451-457, 23 mar, 2016.

STEFFEN, G. P. K.; STEFFEN, R. B.; HANDTE, V. G.; COSTA, A. F. P. Produção de cogumelos comestíveis em substratos orgânicos. Porto Alegre: SEAPDR/DDPA, 2020.

PIAIA, A.; MORAIS, R. M. Produção de cogumelos comestíveis em Substratos orgânicos. Porto Alegre: DDPA, 2020. (Circular: divulgação técnica, n.3).

TEIXEIRA, M. F. S.; PALHETA, R. A.; CASTILlO, T. A. Bioproduto compreendendo micélio, processo de produção do mesmo, e composição alimentícia. Depositante: Fundação Universidade do Amazonas. BR N. PI 1106545-1 A2. Depósito: 04 ago. 2011

TEIXEIRA, M. F. S.; MARTIM, S. R. Produção e extração de coagulante do leite bovino utilizando o cogumelo Pleurotus albidus. Depositante: Fundação Universidade do Amazonas. BR 102017014672-3 A2. Depósito: 06 jul. 2017. 
ZIED, D.C; IOSSI, M.R. Fungicultura: produção de alimentos em pequeno espaço, rápido ciclo de produção e alta tecnologia. Revista Agronomia Brasileira, v. 2, 2018.

Recebido em: 05/01/2022

Aprovado em: 08/02/2022

Publicado em: 12/02/2022 